\title{
Sen2Cor for Sentinel-2
}

Magdalena Main-Knorn, Bringfried Pflug, Jerome Louis, Vincent Debaecker, Uwe Müller-Wilm, et al. 


\title{
Sen2Cor for Sentinel-2
}

\author{
Magdalena Main-Knorn *a , Bringfried Pflug ${ }^{\mathrm{a}}$, Jerome Louis ${ }^{\mathrm{b}}$, Vincent Debaecker ${ }^{\mathrm{b}}$, \\ Uwe Müller-Wilm $^{\mathrm{c}}$, Ferran Gascon ${ }^{\mathrm{d}}$ \\ ${ }^{a}$ German Aerospace Center (DLR), Earth Observation Center, Remote Sensing Technology \\ Institute, Photogrammetry and Image Analysis, 12489 Berlin, Germany; ${ }^{b}$ Telespazio France (TPZ \\ F), SSA Business Unit (Satellite Systems \& Applications), 31023 Toulouse Cedex 1, France; \\ ${ }^{c}$ Telespazio Germany (TPZ V), Telespazio VEGA Deutschland GmbH, Europaplatz 5, 64293

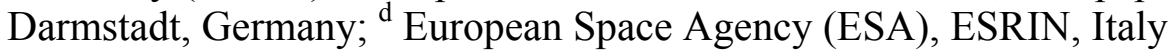

*magdalena.main-knorn@dlr.de; phone +493067055654; fax +493067055642; www.DLR.de/eoc

\begin{abstract}
In the frame of the Copernicus programme, ESA has developed and launched the Sentinel-2 optical imaging mission that delivers optical data products designed to feed downstream services mainly related to land monitoring, emergency management and security. The Sentinel-2 mission is the constellation of two polar orbiting satellites Sentinel-2A and Sentinel-2B, each one equipped with an optical imaging sensor MSI (Multi-Spectral Instrument). Sentinel-2A was launched on June $23^{\text {rd }}, 2015$ and Sentinel-2B followed on March $7^{\text {th }}, 2017$. With the beginning of the operational phase the constellation of both satellites enable image acquisition over the same area every 5 days or less. To use unique potential of the Sentinel-2 data for land applications and ensure the highest quality of scientific exploitation, accurate correction of satellite images for atmospheric effects is required. Therefore the atmospheric correction processor Sen2Cor was developed by Telespazio VEGA Deutschland GmbH on behalf of ESA. Sen2Cor is a Level-2A processor which main purpose is to correct single-date Sentinel-2 Level-1C Top-Of-Atmosphere (TOA) products from the effects of the atmosphere in order to deliver a Level-2A Bottom-Of-Atmosphere (BOA) reflectance product. Additional outputs are an Aerosol Optical Thickness (AOT) map, a Water Vapour (WV) map and a Scene Classification (SCL) map with Quality Indicators for cloud and snow probabilities. Telespazio France and DLR have teamed up in order to provide the calibration and validation of the Sen2Cor processor. Here we provide an overview over the Sentinel-2 data, processor and products. It presents some processing examples of Sen2Cor applied to Sentinel-2 data, provides up-to-date information about the Sen2Cor release status and recent validation results at the time of the SPIE Remote Sensing 2017.
\end{abstract}

Keywords: Sentinel-2, Sen2Cor, atmospheric correction, BOA, AOT, WV, classification, cloud screening

\section{INTRODUCTION}

In the frame of the Copernicus Programme (European initiative for the implementation of information services dealing with environment and security), ESA is developing the space segment with a series of satellite missions, known as Sentinels [1]. Sentinel missions 1 to 3 are designed as a constellation of two satellites each to improve revisit and global coverage requirements. Both radar- and multi-spectral imaging instruments are applied, to monitor land, ocean and atmosphere. Sentinel-1 carries an advanced radar instrument, built on SAR systems heritage (ERS-1, ERS-2, Envisat and Radarsat), providing an all-weather, day-and-night supply of imagery of Earth's surface. Numerous services can benefit from the mission e.g. the monitoring and mapping of sea-ice, monitoring of the marine environment (oil-spill, ship detection), monitoring of land surface motion risks [2]. Sentinel-3 main objective is to measure sea-surface topography, sea- and land-surface temperature and ocean- and land-surface colour with high-end accuracy and reliability in support of ocean forecasting systems, and for environmental and climate monitoring [3]. Sentinel-3 builds directly on a proven heritage of ERS-2 and Envisat, and includes an innovative instrument package of a Sea and Land Surface Temperature Radiometer (SLSTR), an Ocean and Land Colour Instrument (OLCI), and a dual-frequency (Ku and $\mathrm{C}$ band) advanced

Image and Signal Processing for Remote Sensing XXIII, edited by Lorenzo Bruzzone, Francesca Bovolo Proc. of SPIE, Vol. 10427, 1042704 - @ 2017 SPIE · CCC code: 0277-786X/17/\$18 · doi: 10.1117/12.2278218 
Synthetic Aperture Radar Altimeter (SRAL) [4]. The Sentinel-4 and Sentinel-5 missions are dedicated to monitor the composition of the atmosphere for Copernicus Atmosphere Services, including the monitoring of air quality, stratospheric ozone and solar radiation, and climate monitoring [5]. Both missions will be carried on meteorological satellites operated by Eumetsat. Finally, Sentinel-6 mission is designed to complement ocean information from Sentinel3 , and carries a radar altimeter to provide high-precision and timely observations of the topography of the global ocean [6].

\subsection{Sentinel-2 mission}

Sentinel-2 (S-2) is a polar-orbiting, optical mission for land and coastal region monitoring and emergency services, designed with an enhanced spectral range and performances compared to previous similar imaging missions as SPOT and Landsat [7]. The Sentinel-2 mission is dedicated to monitor agriculture, forests, land-use change, land-cover change, mapping biophysical variables such as leaf chlorophyll content, leaf water content, leaf area index, monitoring coastal and inland waters, as well as risk and disaster mapping [8]. The Sentinel-2 mission is the constellation of two satellites Sentinel-2A and Sentinel-2B, each one equipped with an optical imaging sensor MSI (Multi-Spectral Instrument). The MSI aims at measuring reflected radiance in 13 spectral bands spanning from the Visible and Near Infrared (VNIR) to the Short Wave Infra-Red (SWIR) spectral range (Figure.1). Sentinel-2A was launched on June 23rd, 2015 and Sentinel2B followed on March 7th, 2017. With the beginning of the operational phase (June, 16 ${ }^{\text {th }} 2017$ ) the constellation of both satellites enables image acquisition over the same area every 5 days or less, with a 10:30 A.M. descending node.

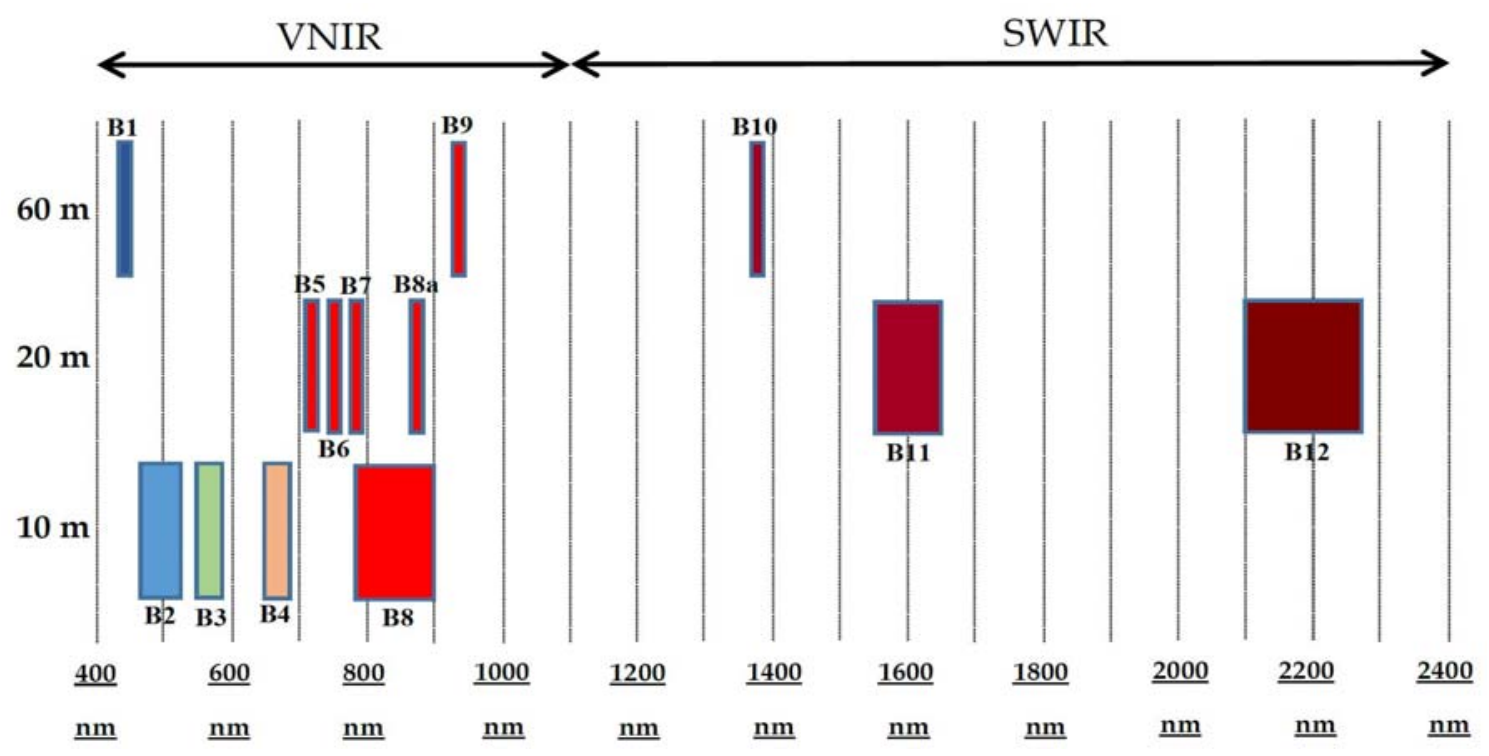

Figure 1. MSI spectral bands vs. spatial resolution with corresponding FullWidth at Half Maximum (FWHM). Source: [9]

To ensure the highest quality of service for the Sentinel-2 mission, ESA has set up the S-2 Mission Performance Centre (MPC) [10]. The MPC is in charge of the overall performance monitoring of the S-2 mission within the S-2 Payload Data Ground Segment (PDGS). It is constituted of a Coordinating Centre (MPC/CC) in charge of the main routine activities, engineering support to the mission, overall service management, and the Expert Support Laboratories (ESLs) providing the scientific expertise according to their area of competency. The S-2 MPC includes five ESLs responsible for processors calibration and validation of Level-1 and Level-2A products. In particular, the ESLs are in charge of the following activities: 1) Multi-spectral instrument (MSI) configuration, 2) L1 and L2A algorithms evolution, verification and maintenance over the mission lifetime, 3) Maintaining the processing baseline, 4) Calibration of the MSI sensor and L1 processor, 5) Radiometric and Geometric validation of L1 products, 6) Calibration of the L2A processor (Sen2Cor), and 7) Geophysical validation of all the L2A products. 


\section{SEN2COR PROCESSOR}

To use unique potential of the Sentinel-2 data for quantitative land applications and ensure the highest quality of scientific exploitation, accurate correction of satellite images for atmospheric effects is required. Therefore the atmospheric correction processor Sen2Cor was developed by Telespazio VEGA Deutschland GmbH on behalf of ESA. Sen2Cor is a Level-2A processor which main purpose is to correct single-date Sentinel-2 Level-1C Top-Of-Atmosphere (TOA) products from the effects of the atmosphere in order to deliver a Level-2A Bottom-Of-Atmosphere (BOA) reflectance product. Additional outputs are an Aerosol Optical Thickness (AOT) map, a Water Vapour (WV) map and a Scene Classification (SCL) map with Quality Indicators for cloud and snow probabilities. Sen2Cor outputs are provided for spatial resolutions $60 \mathrm{~m}, 20 \mathrm{~m}$ and $10 \mathrm{~m}$. Today, Level-2A products can be generated by users with the Sen2Cor processor available as a third-party plugin of the Sentinel-2 Toolbox [11]. Though, L2A data production run on the systematic basis over Europe and dissemination through the Copernicus Open Access Hub [12] started in May 2017.

The basic framework of the Sen2Cor processor (Figure 2) consists of five modules coordinating interactions within the workflow, reading and processing data, providing the configuration parameters, as well as converting the products into the destined format. Two main modules are designed to process the S-2 Level-1C input data, the Scene Classification (SCL) module and the Atmospheric Correction (AC) module. The main processing workflow consists of reading and processing the input parameters and data and the creation of an internal temporary database, which is then used by the $\mathrm{SC}$ and the $\mathrm{AC}$ modules to retrieve and to store the data and intermediate products. The processing can act in a loop, dependent on the number of different product resolutions to be generated. The actual description of the Sen2Cor retrieval methods is published in [13].

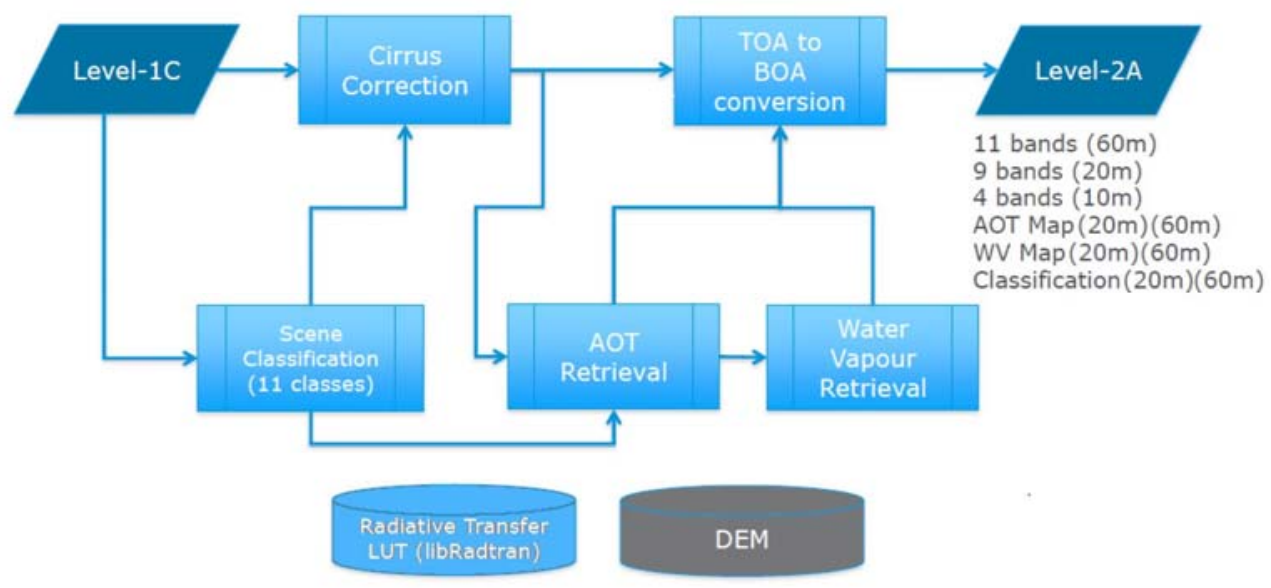

Figure 2. Level-2A processing schema with Sen2Cor. Source: [9]

Sen2Cor can be configured through the L2A_GIPP.xml file. Sen2Cor enables parallel processing of different granules and the number of processes can be set by the user or automatically determined by the processor. Processing procedure allows using a Digital Elevation Model (DEM), provided by the user in DTED format or downloaded automatically by the processor (SRTM). If no DEM is used the mean elevation should be carefully set. The ozone content is selected automatically by Sen2Cor if ECMWF auxiliary data are present in the L1C-input data.

\subsection{The Scene Classification (SCL)}

The SCL algorithm allows to detect clouds, their shadows and snow, and to generate a classification map. The latter consists of 11 classes, including 2 classes for cloud probabilities (medium, and high), thin cirrus class, vegetation, soils/deserts, water, snow, dark area pixels, cloud shadows, and unclassified, saturated or defective pixels. This map is used internally in Sen2Cor in the AC module to distinguish between cloudy, clear and water pixels, but it does not perform a land cover classification map in a strict sense [9]. The algorithm is based on a series of threshold tests that use as input top-of-atmosphere reflectance from the Sentinel-2 spectral bands. In addition, thresholds are applied on band ratios and indices like the Normalized Difference Vegetation (NDVI) and Snow Index (NDSI). For each of these 
thresholds tests, a level of confidence is associated. At the end of the processing chain a probabilistic cloud mask quality map and a snow mask quality map are produced. The algorithm uses the reflective properties of scene features to establish the presence or absence of clouds in a scene. Cloud screening is applied to the data in order to retrieve accurate atmospheric and surface parameters as input for further processing steps, either the ones described below or higher-level processing such as mosaicking or time-series analysis.

\subsection{The Atmospheric Correction (AC)}

The AC is performed using a set of Look-Up tables generated via libRadtran [14]. Baseline processing is the rural/continental aerosol type. More Look-Up tables enable processing with the choice between midlatitude winter and midlatitude summer atmosphere, rural and maritime aerosol and ozone content according to the scene geographic location and climatology. The AC module is a porting and adaptation of the ATCOR software [15] (Satellite version tailored to Sentinel-2, Dec 2010, written in IDL) into Python, and encompasses following corrections:

Aerosol Optical Thickness (AOT): AOT retrieval provides a measure for the visual transparency of the atmosphere. It is derived at 550nm using the DDV (Dense Dark Vegetation) algorithm [16], based on the correlation between reflectance in SWIR (band 12) and VIS (red - band 4, and blue - band 2). The algorithm requires that the scene contains reference areas of known reflectance behaviour, preferably DDV and/or dark soil and water bodies. If the scene contains no DDV pixels, the fallback solution is to perform the atmospheric correction with a constant AOT which is specified by the start visibility (VIS) set in the configuration file [13]. Default value of start visibility is $40 \mathrm{~km}$ which corresponds to an AOT at $550 \mathrm{~nm}$ of 0.2 at sea level. The quantification value to convert Digital Numbers to AOT is equal to 1000. The algorithm delivers an AOT map.

Water Vapour: WV retrieval over land is performed with the Atmospheric Pre-corrected Differential Absorption algorithm [17] which is applied to the two Sentinel-2 bands B8a, and B9. Band 8a is the reference channel in an atmospheric window region. Band B9 is the measurement channel in the absorption region. The absorption depth is evaluated by calculating the radiance for an atmosphere with no water vapour, assuming that the surface reflectance for the measurement channel is the same as for the reference channel. The absorption depth is then a measure of the water vapour column content. The quantification value to convert Digital Numbers to water vapour column in $\mathrm{cm}$ is equal to 1000 .

Additionally, the Sen2Cor processor includes several optional corrections that can be activated like cirrus correction, terrain correction, adjacency correction and empirical Bidirectional Reflectance Distribution Function (BRDF) corrections. While Sen2Cor was developed for applications over land, it can be also applied over water surface using the AOT estimated over land pixels in the image. However the processor contains no consideration of water surface effects like sun glint.

The final Sen2Cor surface reflectance images are provided at different spatial resolutions for following spectral bands:

- 10m resolution for the native bands: B02, B03, B04 and B08;

- 20m resolution for the resampled bands: B02, B03, B04, and the native bands: B05, B06, B07, B8A, B11 and B12;

- 60m resolution for the native bands: B01 and B09, and the resampled bands: B02, B03, B04, B05, B06, B07, B8A, B11 and $\mathrm{B} 12$.

Band B10 is not provided in surface reflectance as the output of Sen2Cor, because it does not provide information on the surface but on the cirrus clouds.

The BOA values are coded in JPEG2000 with the quantification value of 10,000 , i.e., a factor of $1 / 10,000$ needs to be applied to Level-2A digital numbers $(\mathrm{DN})$ to retrieve physical surface reflectance values.

\section{THE LEVEL-2A CALIBRATION AND VALIDATION}

Telespazio France and DLR have teamed up in order to provide the calibration and validation of the Sen2Cor processor. The Level-2A Calibration and Validation activities encompass two main domains: radiometry, which concerns the surface reflectance, water vapour and aerosol outputs of Sen2Cor, and classification, which involves Cloud Screening and Classification outputs. Calibration and Validation datasets cover different atmospheric conditions and different 
latitudes in order to cover various solar angles and seasons, as well are representative for all continents, different topography and land cover types like cropland, forest, grass, sparsely vegetated land, urban, coastal, wetlands, lakes, desert and snow.

Figure 3 gives an example of the Sen2Cor outputs for selected test site over Barrax (Spain), processed with default configuration. Comparing the L1C TOA and the L2A BOA images the latter is perceptively more clear. The SCL image reveals correctly classified vegetation, not-vegetated and clouds pixels, whereas cloud shadows are partly identified as dark area pixels. The AOT and WV images appear very heterogeneous, while it is due to the stretching effects not the value range.

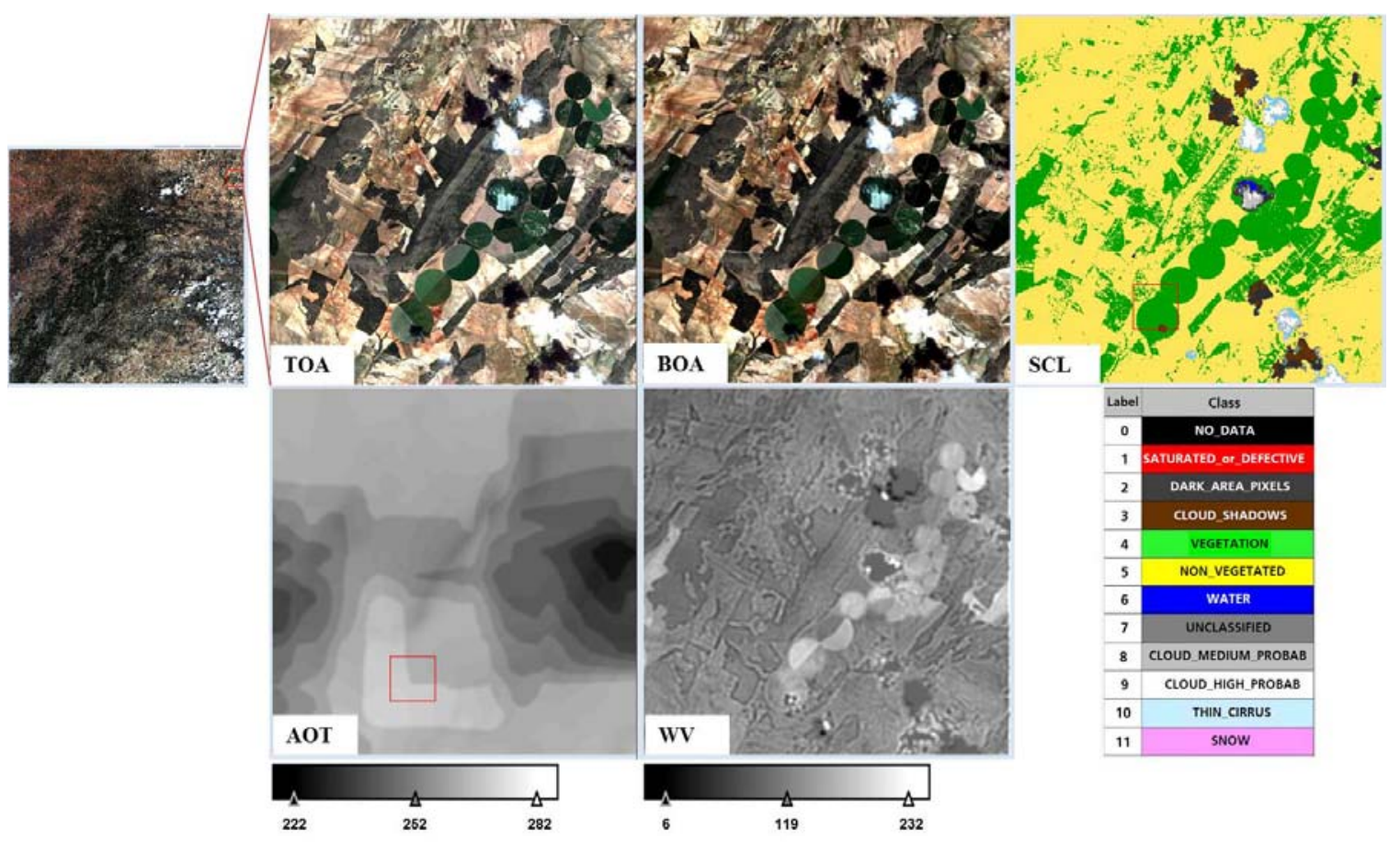

Figure 3. Example of the Sen2Cor outputs for one selected test site over Barrax (Spain), acquired on May, 19th 2017.

\subsection{L2A Calibration Procedure}

Calibration of radiometry outputs focuses on the Atmospheric Correction parametrization and comprises following activities:

- Investigation of the processor sensitivity to the parameters stored in the processor configuration files (e.g. thresholds). Several runs of Sen2Cor are performed with modification of single calibration parameter of interest (e.g. minimum DDV area, Aerosol type ratio threshold, BRDF lower bound). The impact of the individual parameter variations on the different L2A products is analyzed and compared to the reference data, originate from the AERONET network;

- Qualitative analysis of the impact of the activation/deactivation of yes/no parameter is undertaken (e.g. cirrus correction, BRDF correction). These choices usually depend on the particular kind of user applications or scene landscape. Therefore the individual impact of each parameter is assessed on a variety of landscapes and weather conditions.

Calibration of Cloud Screening and Classification algorithm is based on an empirical approach and encompasses full calibration of threshold parameters. The calibration procedure consists of:

- Run of Sen2Cor processor using default thresholds to produce SC products; 
- Manual inspection of pixel classification results to superimpose the scene classification map and L1C spectral bands. The outputs of this step are a performance assessment of the classification for each class reporting on: over-detection, under-detection, misclassification (indicating the wrong class assigned) and how the edges/boundaries between classes are handled by the processor;

- Based on this first assessment, a list of potential improvements is identified with their corresponding thresholds. The thresholds linked to thin clouds are one of the most difficult to find, because in some cases their tuning lead to over detection and in other cases to under detection;

- The proper tuning activity consists in manually slightly varying the SC thresholds to improve the SCL;

- $\quad$ Run of Sen2Cor processor using a new processing baseline (set of updated SCL parameters);

- A quantitative comparison exercise performed between the results of the updated baseline and the results of the standard baseline to assess the impact of the algorithm, i.e. the absolute and relative variation of the number of pixels per class, the class origin for the new classified pixels.

\subsection{L2A Validation Procedure}

Validation provides an accuracy assessment of the SCL product and radiometric validation of WV, AOT and BOA products. Both, AOT and WV are critical parameters for atmospheric correction and have a large influence on the accuracy of BOA [9]. SCL validation is performed on full granules with area of $110 \mathrm{~km} \mathrm{x} 110 \mathrm{~km}$ whereas radiometric validation is performed on area of $9 \mathrm{~km} \times 9 \mathrm{~km}$ around sun photometer location.

Validation of SCL consists of verification of the cloud screening and classification accuracy to reduce L2A products uncertainties. The SCL validation is limited due to the lack of "ground truth" data sets. Therefore, validation of classification map relies on visual inspection, supplemented by meteorological data, if available. Validation is performed using a set of test sites (Figure 4) representative for a large range of land cover types and weather condition (e.g. sun position, elevation, cloud cover). The SCL involves the following steps:

- Selection of a reference database based on a stratified random sampling (minimum of 50 samples per class), in order to guarantee statistical consistency (validity), and avoid exclusion of spatially limited classes from the validation;

- Visual pixel-based inspection of the samples, supported by RGB band composite (Bands 4,3,2), Colour InfraRed composite to highlight vegetation features (bands 8A,4,3) or snow (bands 12, 11,8A), spectral profile, snow and cloud confidence Quality Images, and L1C cirrus band (B10);

- Either one pixel or an area containing pixels of the same class can be labelled; all labelled pixels are used to create the reference classification image;

- Comparison between Sen2Cor SCL output and reference classification;

- Calculation of an error matrix and accuracy statistics.

Validation of WV, AOT and BOA products is performed for the same test sites and in-situ data sets (Figure 4). Validation of AOT and WV products is based on a direct comparison of Sen2Cor outputs with ground reference from AERONET sunphotometer measurements [18] and is performed as follow:

- Interpolation of AOT at $550 \mathrm{~nm}$ and WV content to Sentinel-2 overpass time after successful download of AERONET data;

- Processing of Sentinel-2 L1C products to L2A products using the default Sen2Cor configuration and with SRTM-DEM activated;

- Extraction of a spatial subset of $9 \mathrm{~km} \mathrm{x} 9 \mathrm{~km}$ around sunphotometer location from L2A output, and comparison of the averaged values with the reference. Note that for AOT validation the average of all pixels within $81 \mathrm{~km}^{2}$ window are considered, while for WV validation water pixels are excluded.

Validation of BOA reflectance products is performed by comparing Sen2Cor outputs with BOA reference data computed by a second run of Sen2Cor using AOT550 information provided by collocated sunphotometer measurements as input. 
The resulting "sunphotometer-corrected" BOA data are considered to provide the surface reflectance "truth", since the greatest uncertainty in atmospheric correction comes from the aerosol characterization.

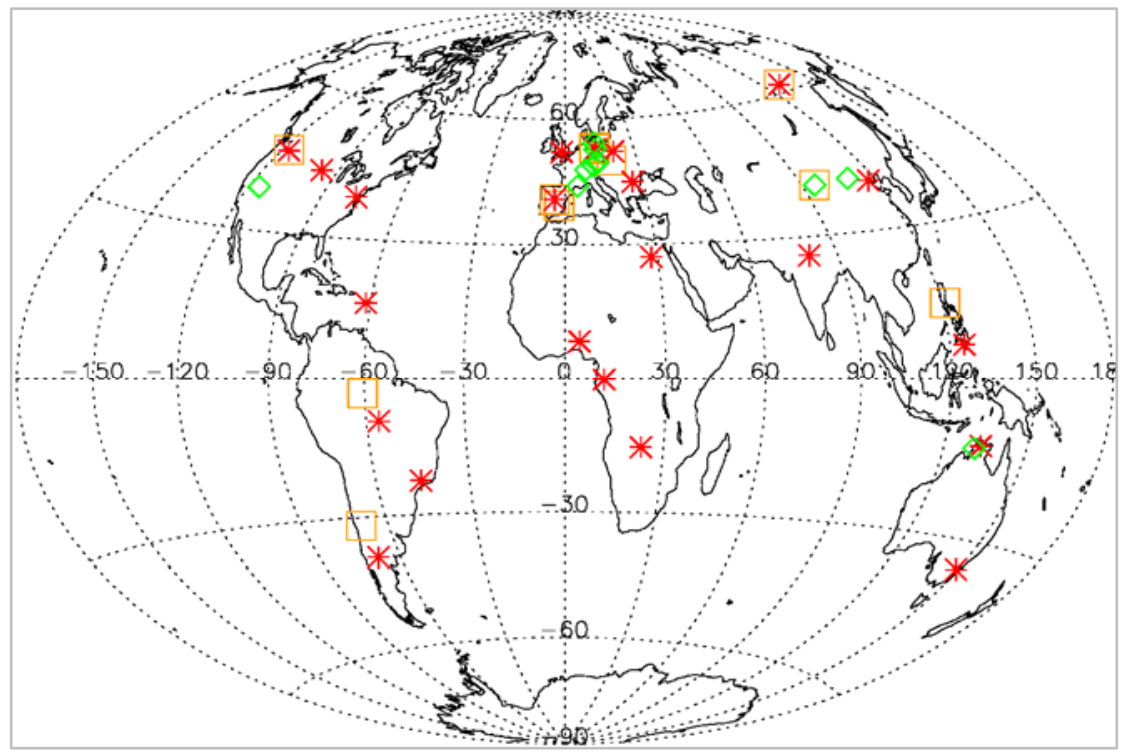

Figure 4. Geographical distribution of the selected core and back-up test sites for the L2A Validation (Red asterisks: Test sites with sunphotometers for AOT, WV and BOA validation; Green diamonds: Test sites for reference surface reflectance measurements; Orange squares: Test sites for SCL validation).

\section{SEN2COR VALIDATION EXAMPLES}

This chapter presents some examples of Sen2Cor L2A products processed with the Sen2Cor Version 2.3.1 and recent validation results at the time of the SPIE Remote Sensing 2017. Since the processing baseline evolves to take into account corrections of errors in the processing chain and introductions of new product features or processing steps, the improved version of Sen2Cor 2.4.0 is already available.

\subsection{SCL Validation results}

Reference classification images were created and compared to corresponding pixels of the Sen2Cor classification images for seven scenes over Barrax (Spain), Berlin (Germany), Yakutsk (Russia), Casleo (Argentina), and Antarctic. One example of data analysis is shown in Figure 5.
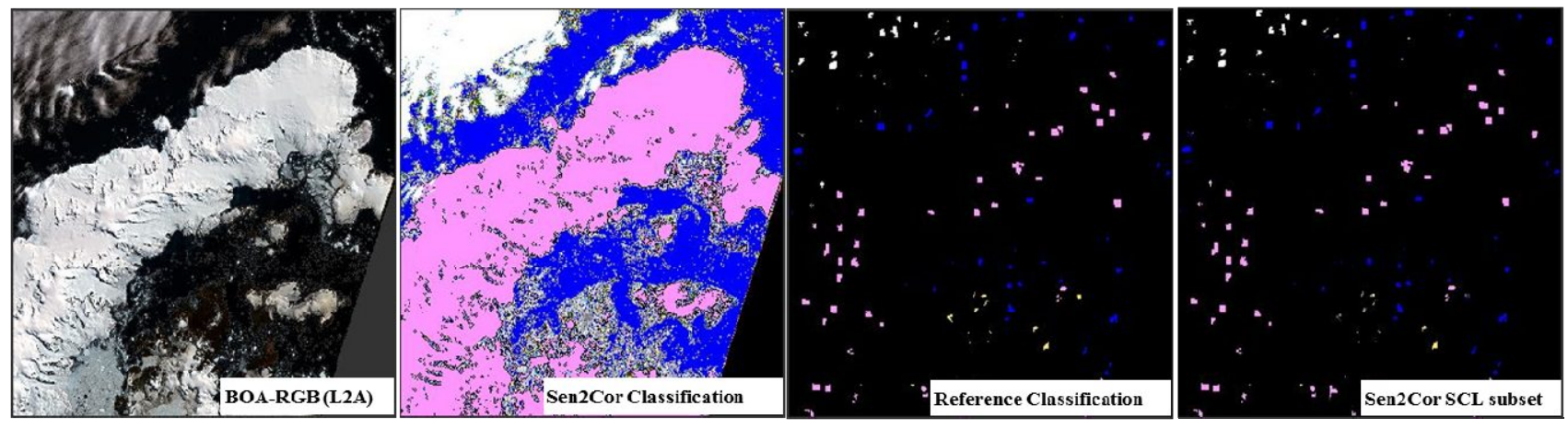

Figure 5. Example image for Sen2Cor SCL product over Antarctic test site, acquired on February 4, 2016. This example represents various topography (flat and rough) and complex weather conditions (presence of snow and clouds). 
The image contains thin clouds over water and land, and is covered partially by snow. Table 1 contains the corresponding confusion matrix. The diagonal in bold shows the number of correctly classified pixels in the validation set. The resulting user's accuracy (UA) indicates the probability that the pixel was classified correctly, while producer's accuracy (PA) reflects the probability that a pixel belongs to the class classified by Sen2Cor.

Table 1. Example of confusion matrix for classification validation over Antarctic test site, with the resulting user's accuracies (UA) and producer's accuracies (PA).

\begin{tabular}{|c|c|c|c|c|c|c|c|c|c|c|c|c|c|}
\hline \multirow[b]{2}{*}{ Classification } & \multicolumn{12}{|c|}{ Reference } & \multirow[t]{2}{*}{ UA (\%) } \\
\hline & (1) & (2) & (3) & (4) & (5) & (6) & (7) & (8) & (9) & $(10)$ & (11) & Sum & \\
\hline (1) Saturated or defective & $\mathbf{0}$ & 0 & 0 & 0 & 0 & 0 & 0 & 0 & 0 & 0 & 0 & 0 & none \\
\hline (2) Dark area pixels & 0 & 30 & 0 & 0 & 5204 & 1 & 1071 & 166 & 0 & 0 & 2 & 6474 & 0.46 \\
\hline (3) Cloud shadows & 0 & 8 & $\mathbf{0}$ & 0 & 310 & 0 & 945 & 149 & 0 & 0 & 0 & 1412 & 0.00 \\
\hline (4) Vegetation & 0 & 0 & 0 & $\mathbf{0}$ & 0 & 0 & 0 & 0 & 0 & 0 & 0 & 0 & none \\
\hline (5) Not-vegetated & 0 & 0 & 0 & 0 & 8645 & 0 & 0 & 0 & 0 & 0 & 0 & 8645 & 100.00 \\
\hline (6) Water & 0 & 117 & 0 & 0 & 2159 & 142521 & 1315 & 416 & 0 & 0 & 24 & 146552 & 97.25 \\
\hline (7) Unclassified & 0 & 8 & 0 & 0 & 8637 & 0 & 74 & 1289 & 71 & 0 & 40 & 10119 & 0.73 \\
\hline (8) Cloud medium probability & 0 & 9 & 0 & 0 & 870 & 1 & 13 & 1922 & 867 & 0 & 0 & 3682 & 52.20 \\
\hline (9) Cloud high probability & 0 & 0 & 0 & 0 & 6 & 0 & 0 & 916 & 57401 & 0 & 0 & 58323 & 98.42 \\
\hline (10) Thin cirrus & 0 & 250 & 0 & 0 & 0 & 0 & 0 & 0 & 0 & $\mathbf{0}$ & 596 & 846 & 0.00 \\
\hline (11) snow & 0 & 3 & 0 & 0 & 98 & 4 & 2 & 1346 & 1024 & 0 & 297390 & 299867 & 99.17 \\
\hline Sum (pixels in reference) & 0 & 425 & 0 & 0 & 25929 & 142527 & 3420 & 6204 & 59363 & 0 & 298052 & 535920 & \\
\hline PA (\%) & none & 7.06 & none & none & 33.34 & 100 & 2.16 & 30.98 & 96.69 & none & 99.78 & & \\
\hline
\end{tabular}

Considering this example product over Antarctic water, snow and cloudy pixels were perfectly recognized by Sen2Cor, with all accuracies over $96 \%$. Clear land pixels (not-vegetated class) were classified correctly, while some regions of the image were not identified as a land and misclassified in favour of dark pixels or unclassified.

The summary statistic over seven Sen2Cor L2A products is presented in Table 2. The analysis considers examples of both Sentinel-2A and Sentinel-2B L1C data over at five locations. The examples represent flat and mountainous test sites, the cloud cover ranges from $2 \%$ to $70 \%$ with the presence of cumulus and thin cirrus clouds, and snow cover.

For evaluation, besides UA and PA, also overall accuracy (OA), commission error (CE) and omission error (OE) are reported. Commission error indicates percentage of class pixels in the classification image which are falsely classified, and equals 100 minus UA. Omission error describes the share of reference pixels in that class that have been "omitted" in the classification image, and equals 100 minus PA.

Generally, the average OA for all classes is $(74 \pm 11) \%$. Considering only the separation between usable (Land-Water) and non-usable (Others) pixels, the performance is undoubtedly higher and OA reaches $(92 \pm 4) \%$. As the main objective of the Sen2Cor SCL module is to distinguish between cloudy pixels, clear pixels and water pixels, Sen2Cor 2.3.1 provides a satisfactory results.

The accuracies of all classes differ from one class to another, and between products. Nevertheless, the highest performance of Sen2Cor can be reported for the classes of cloud high probability pixels, not-vegetated and snow. Classification of dark areas and cloud shadows varies due to the different terrains and cloud types at different altitudes in the atmosphere. The most challenging is the classification of very thin cirrus clouds, which have only little effect on the reflectances in the VIS/NIR channels. 
Table 2. A summary statistics over 7 products (averaged accuracies, commissions and omissions per class).

\begin{tabular}{|c|c|c|c|c|c|}
\hline Sen2Cor Class & user's accuracy & commission error & producer's accuracy & omission error & \\
\hline Saturated or defective & none & none & none & none & \\
\hline Dark area pixels & 2.02 & 83.84 & 15.41 & 84.59 & \\
\hline Cloud shadows & 79.64 & 20,36 & 23.50 & 60.00 & \\
\hline Vegetation & 48.65 & 31.55 & 52.73 & 27.47 & \\
\hline Not-vegetated & 92.62 & 7.38 & 65.39 & 34.61 & \\
\hline Water & 64.52 & 35.49 & 44.66 & 38.84 & \\
\hline Unclassified & 1.78 & 84.07 & 10.99 & 89.01 & \\
\hline Cloud medium probability & 26.99 & 73.02 & 31.44 & 68.56 & \\
\hline Cloud high probability & 80.54 & 5.31 & 88.72 & 11.28 & \\
\hline Thin cirrus & 17.69 & 65.81 & 37.91 & 62.10 & \\
\hline Snow & 46.91 & 53.09 & 92.86 & 7.14 & \\
\hline Overall accuracy & & & & & 73.69 \\
\hline Pixels validated: & & & & & 181532 \\
\hline \multicolumn{6}{|l|}{ Simplified statistics: } \\
\hline Land-Water pixels & 73.78 & 26.22 & 63.19 & 36.81 & \\
\hline Others (non-usable) & 92.69 & 7.31 & 97.35 & 2.65 & \\
\hline Overall accuracy (OA) & & & & & 92.36 \\
\hline
\end{tabular}

\subsection{AOT and WV Validation results}

The Sentinel-2 L1C products were processed to L2A using the default Sen2Cor configuration (e.g., cirrus correction deactivated) and with SRTM DEM activated. AOT and WV validation results are shown in Figure 6.

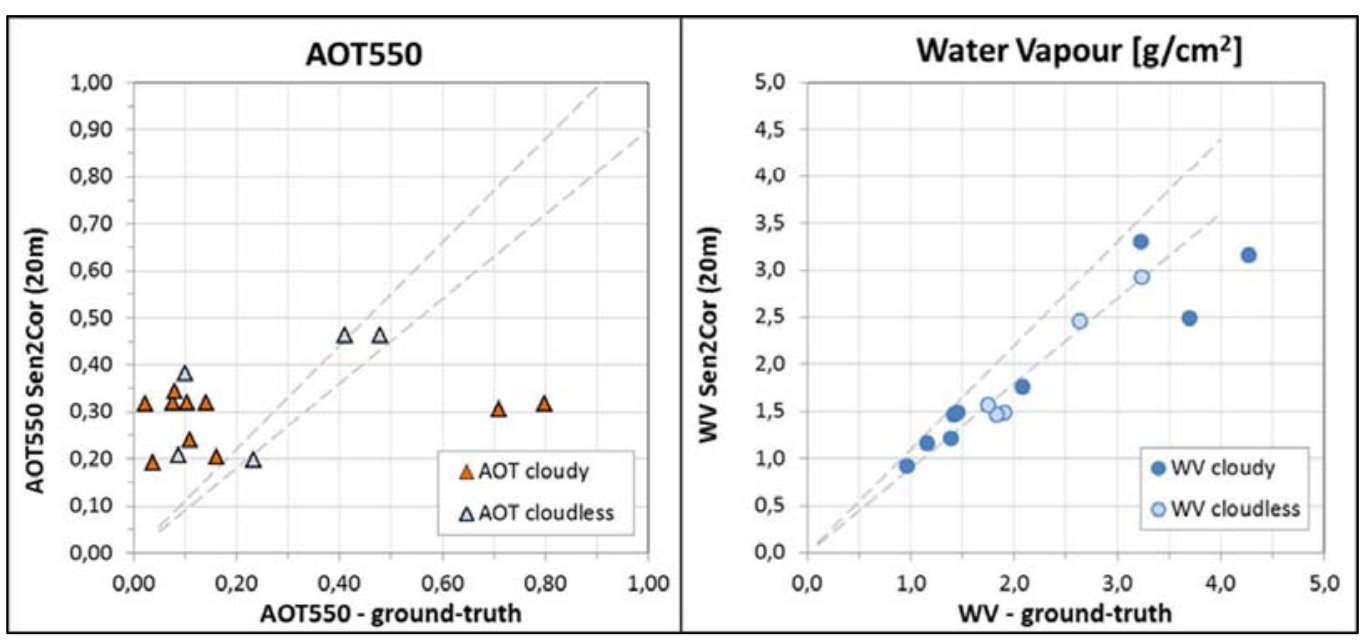

Figure 6. Validation of AOT and WV by direct comparison of Sen2Cor values (average over $9 \mathrm{~km} \mathrm{x} 9 \mathrm{~km}$ ) with ground reference from AERONET. 
For the investigated examples Sen2Cor shows good agreement between Sen2Cor AOT values and ground measurements, while large differences also occurred. The latter was mostly related to the lack of DDV pixels on the image, which are critical for the AOT retrieval algorithm implemented in Sen2Cor. If not sufficient DDV pixels are found, AOT is set to a default value of 0.20 (corresponds to default visibility of $40 \mathrm{~km}$ ).

Considering only examples with sufficient number of DDV pixels and with cloud cover below 5\%, the Sen2Cor performance remarkable increased and a mean difference between Sen2Cor values and ground reference is $0.03 \pm 0.02$ with the maximum difference of 0.05 . This validation results are aligned with the results for other atmospheric correction processors found in the literature $[19,20]$.

Sen2Cor Water Vapour retrieval shows better agreement with ground measurements than AOT retrieval (Figure 6) and cloud cover and missing DDV pixels have less impact on retrieval accuracy. Higher WV values tend to be underestimated by Sen2Cor. Considering again only cloudless examples a mean difference between Sen2Cor and ground measurements reaches $(0.29 \pm 0.11) \mathrm{g} / \mathrm{cm}^{2}$, with maximum difference of $0.42 \mathrm{~g} / \mathrm{cm}^{2}$.

\subsection{BOA Validation examples}

BOA validation is performed on the same test sites and in-situ data sets as the ones used for validation of AOT and WV products. An example of BOA validation over test site Belsk (Poland) for a typical locations with sufficient number of DDV pixels is presented on the Figure 7.
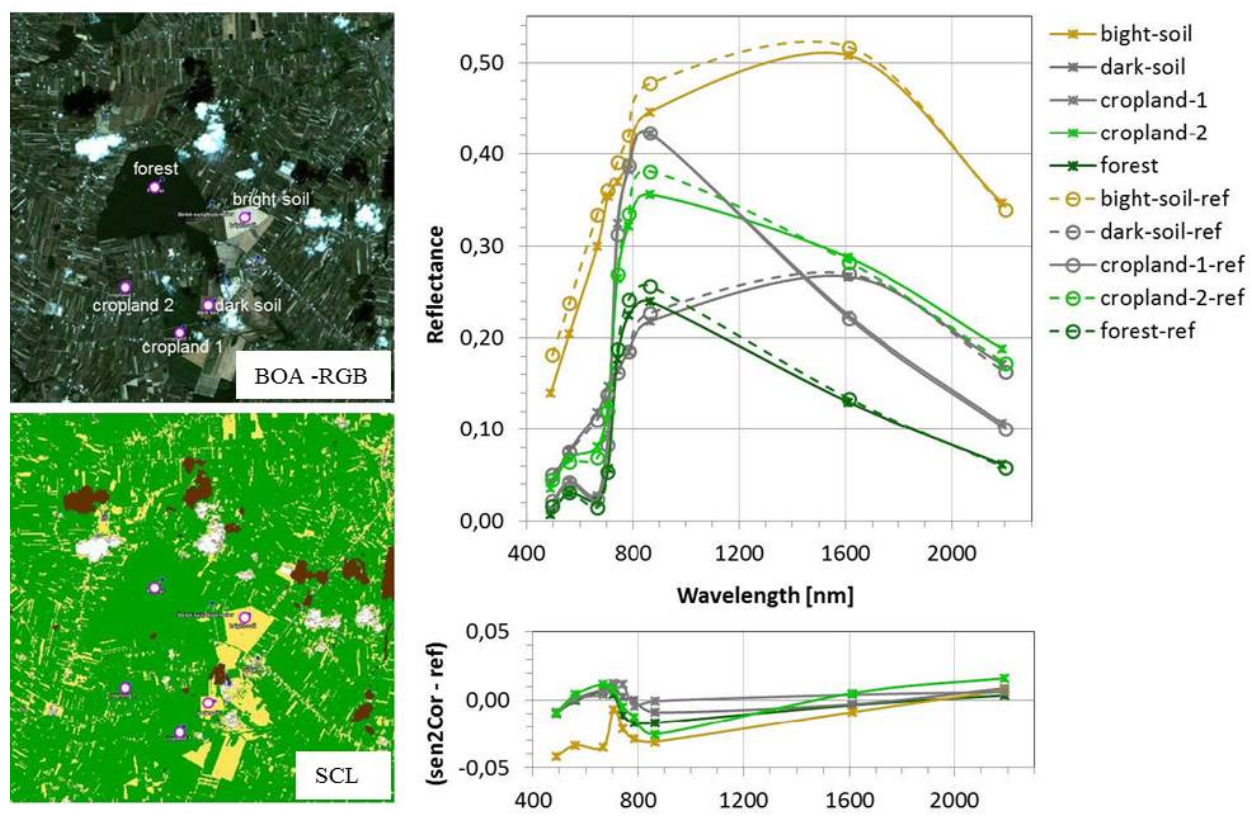

Figure 7. Validation example of BOA reflectance on test site Belsk (Poland). Sentinel-2 image was acquired on August, $14^{\text {th }}$ 2015. Sen2Cor BOA and SCL images represent subsets of $9 \mathrm{~km} \mathrm{x} 9 \mathrm{~km}$ area around AERONET station. Example spectra of soil and vegetation are shown as well as relative difference to reference spectra at indicated locations.

In this example spectra for dark and bright soils, for forest and other vegetation show expected spectral profiles and agree within the target accuracy of relative $5 \%$ for BOA reflectance. The BOA relative difference of 0.04 leads to a Normalized Density Vegetation Index (NDVI) uncertainty up to 0.06 .

Sentinel-2 L2A data processed with Sen2Cor were also compared to atmospherically-corrected Landsat-8 data [21]. The comparison study was conducted on Sentinel-2 and Landsat-8 (L8) images registered on the same day over six test sites in Europe (located in Greece, Turkey, Austria, Germany, Czech Republic, and France). The agreement between Sen2Cor BOA and L8 reached an overall Root Mean Square Error (RMSE) of 0.031 over all six bands used for comparison. This 
study confirms spectral consistency of Sentinel-2 Sen2Cor products with Landsat-8 and with ground reflectance measurements and shows the high potential and quality of Sentinel-2 data for further application.

Other study considered the potential of Sentinel-2 L2A products for water applications even if Sen2Cor was developed as atmospheric correction processor over land surface [22]. In this study Sen2Cor processor was compared with other atmospheric corrections developed particularly for application over water bodies with the spectra measured on a lake. Comparison at seven test points showed that all 3 algorithms give comparable results. RMSE between Sen2Cor BOA reflectance and in-situ measurements ranges from 0.002 to 0.005 .

\section{CONCLUSIONS}

The main objective of the Sentinel-2 MSI mission is to provide stable time series of images at high spatial resolution. With the launch of both Sentinel-2 satellites and the beginning of the operational phase of Sentinel-2B this objective is mainly achieved. Sentinel-2 time series are consistent and comparable with other missions such as Landsat-8 OLI.

For investigated Sen2Cor products, processor provides L2A SCL products with an overall accuracy of (73 \pm 11$) \%$ for all classes and OA of $(92 \pm 4) \%$ for usable/non-usable pixels separation. Uncertainty of AOT retrieval is $0.03 \pm 0.02$ if the image contains sufficient number of DDV pixels. In other case, a processor evolution is being developed using aerosol information from ECMWF. WV retrieval performs better comparing to AOT and the uncertainty is of $(0.29 \pm$ $0.11) \mathrm{g} / \mathrm{cm} 2$. Conversion of Sentinel-2 L1C TOA to L2A BOA reflectance gives uncertainties in surface reflectance up to 0.04 in products with sufficient DDV pixels.

Finally, the Sentinel-2 time series need to meet the high availability, reliability and timeliness performance requirements to support the Copernicus services [9]. Sen2Cor processing baseline evolves to take into account corrections and improvements, and the essentially stabilized new version of Sen2Cor 2.4.0 is already available for users.

\section{ACKNOWLEDGEMENTS}

The authors thank the PI investigators and their staff for establishing and maintaining the AERONET sites used in this investigation.

\section{REFERENCES}

[1] E. Commission, [Final - Impact Assessment accompanying the document "Proposal for a Regulation of the European Parliament and of the Council establishing the Copernicus Programme and repealing Regulation (EU) No 911/2010], Brussels(2013).

[2] E. Attema, P. Borgellini, P. Edwards et al., "Sentinel-1 - The radar mission for GMES land and sea services," Esa Bulletin-European Space Agency(131), 10-17 (2007).

[3] M. Aguirre, B. Berruti, J. L. Bezy et al., "Sentinel-3 - The ocean and medium-resolution mission for GMES operational services," Esa Bulletin-European Space Agency(131), 24-29 (2007).

[4] Y. Le Roy, M. Deschaux-Beaume, C. Mavrocordatos et al., [SRAL SAR radar altimeter for sentinel-3 mission], (2007).

[5] P. Ingmann, B. Veihelmann, J. Langen et al., "Requirements for the GMES Atmosphere Service and ESA's implementation concept: Sentinels-4/-5 and-5p," Remote Sensing of Environment, 120, 58-69 (2012).

[6] ESA, [Sentinel-6 (jason - cs)], April 2015, http://esamultimedia.esa.int/docs/EarthObservation/Sentinel6 facts 2015.pdf (11 July 2017).

[7] M. Drusch, U. Del Bello, S. Carlier et al., "Sentinel-2: ESA's Optical High-Resolution Mission for GMES Operational Services," Remote Sensing of Environment, 120, 25-36 (2012). 
[8] ESA, [Sentinel-2: ESA's Optical High-Resolution Mission for GMES Operational Services (ESA SP-1322/2 March 2012)] ESA Communications, ESTEC, PO Box 299, 2200 AG Noordwijk, The Netherlands(2012).

[9] F. Gascon, C. Bouzinac, O. Thépaut et al., "Copernicus Sentinel-2A Calibration and Products Validation Status," Remote Sensing, 9(6), 584 (2017).

[10] M. Berger, J. Moreno, J. A. Johannessen et al., "ESA's sentinel missions in support of Earth system science," Remote Sensing of Environment, 120, 84-90 (2012).

[11] [Sentinel-2 Toolbox. Available online: http://step.esa.int/main/toolboxes/sentinel-2-toolbox (accessed on 6 December 2016).].

[12] [Copernicus Open Access Hub. Available online: https://scihub.copernicus.eu/ (accessed on 22 May 2017).].

[13] R. Richter, J. Louis, and U. Müller-Wilm, [Sentinel-2 MSI - Level 2A Products Algorithm Theoretical Basis Document; S2PAD-ATBD-0001, Issue 2.0] Telespazio VEGA Deutschland GmbH, Darmstadt, Germany(2012).

[14] B. Mayer, and A. Kylling, "Technical note: The libRadtran software package for radiative transfer calculations description and examples of use," Atmos. Chem. Phys., 5(7), 1855-1877 (2005).

[15] R. Richter, D. Schlapfer, and A. Muller, "An automatic atmospheric correction algorithm for visible/NIR imagery,” International Journal of Remote Sensing, 27(9-10), 2077-2085 (2006).

[16] Y. J. Kaufman, and C. Sendra, "Algorithm for Automatic Atmospheric Corrections to Visible and near-Ir Satellite Imagery,” International Journal of Remote Sensing, 9(8), 1357-1381 (1988).

[17] D. Schläpfer, C. C. Borel, J. Keller et al., "Atmospheric Precorrected Differential Absorption Technique to Retrieve Columnar Water Vapor," Remote Sensing of Environment, 65(3), 353-366 (1998).

[18] B. N. Holben, T. F. Eck, I. Slutsker et al., "AERONET-A Federated Instrument Network and Data Archive for Aerosol Characterization," Remote Sensing of Environment, 66(1), 1-16 (1998).

[19] B. Pflug, M. Main-Knorn, A. Makarau et al., "Validation of aerosol estimation in atmospheric correction algorithm ATCOR,” Int. Arch. Photogramm. Remote Sens. Spatial Inf. Sci., XL-7/W3, 677-683 (2015).

[20] F.-M. Bréon, A. Vermeulen, and J. Descloitres, "An evaluation of satellite aerosol products against sunphotometer measurements," Remote Sensing of Environment, 115(12), 3102-3111 (2011).

[21] F. Vuolo, M. Żółtak, C. Pipitone et al., "Data Service Platform for Sentinel-2 Surface Reflectance and ValueAdded Products: System Use and Examples,” Remote Sensing, 8(11), 938 (2016).

[22] K. Dörnhöfer, A. Göritz, P. Gege et al., "Water Constituents and Water Depth Retrieval from Sentinel-2A-A First Evaluation in an Oligotrophic Lake," Remote Sensing, 8(11), 941 (2016). 